\title{
Developing, Maintaining, and Teaching Clinical Diagnostic Expertise
}

Bruce Fisher MD, and Liam Rourke, PhD

\section{About the Authors}

Bruce Fisher is a general internist and educator at the University of Alberta in Edmonton, Alberta, Canada. His interests include teaching and assessing clinical examination skills and clinical reasoning, and peer-review for clinical teaching. Liam Rourke is an educational psychologist at the University of Alberta, who is interested in perceptual learning in medicine. Dr. Rourke's research is funded by grants from the Social Sciences and Humanities Research Council, the MSI Foundation, and the University Hospital Foundation.

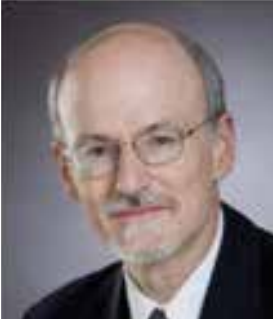

Fisher

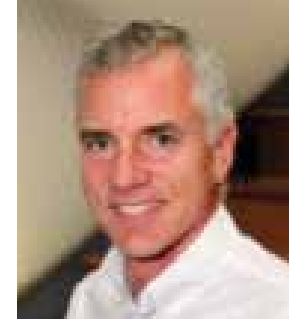

Rourke

\section{Summary}

Understanding the process of expert clinical reasoning improves our ability to develop, practice, maintain, teach, and assess clinical diagnostic expertise. The dual process model describes a synergistic interplay of associative thinking and analytical reasoning. These complimentary processes facilitate the efficient abstraction of data from clinical presentations, the identification of key features, and the production of useful problem representations. These are compared unconsciously to prototypical cases stored in memory as illness scripts for a best match. A lack of a satisfactory match may stimulate a conscious, analytic analysis of discordance, ideally reducing bias and error and promoting further script development.

An awareness of this process and the use of existing observation and assessment techniques can enable both the teaching and the assessment of clinical reasoning. Learners can also be taught to use these techniques to help develop self-assessment of clinical reasoning performance. Teaching and assessing clinical reasoning in others stimulates clinician teachers to reflect on their own clinical reasoning and practice, serving as an effective form of continuous professional learning.

\section{Résumé}

Une bonne compréhension du processus de raisonnement clinique spécialisé améliore la capacité de développer, de mettre en pratique, de maintenir, d'enseigner et d'évaluer l'expertise clinique en matière de diagnostic. Le modèle à double processus décrit l'interaction synergique de la pensée associative et du raisonnement analytique. Ces processus complémentaires permettent de développer un modèle abstrait efficient à partir de données tirées de cas cliniques, de découvrir les caractéristiques clés, ainsi que de produire des modèles de problématiques qui s'avèrent fort utiles. Ces derniers se comparent inconsciemment aux cas soumis comme prototypes et emmagasinés dans la mémoire comme modèles de cas, et ce, en vue d'établir la meilleure correspondance possible. L'absence d'une correspondance satisfaisante peut stimuler une analyse consciente et critique de la dissonance et, idéalement, mener à réduire les distorsions et les erreurs, ainsi qu'à favoriser l'émergence de nouveaux modèles.

La conscientisation de ce processus jumelée au recours à des techniques d'observation et d'évaluation peut permettre à la fois l'enseignement et l'évaluation du raisonnement clinique. On peut également enseigner aux apprenants à utiliser ces outils pour favoriser l'auto-évaluation de l'efficacité de leur raisonnement clinique. Par ailleurs, le fait d'enseigner et d'évaluer le raisonnement clinique amène les enseignants cliniciens à réfléchir sur leurs propres pratiques et raisonnement clinique, et constitue ainsi une forme efficace de formation professionnelle continue. 
A s practising General Internists, most of us would agree Athat the acquisition of clinical diagnostic expertise is a painstaking, slow and experiential process. We may not so readily agree about the nature and effectiveness of methods used to make this happen, and such inquiry and debate continues to be the stuff of major medical education research. However, there is a growing consensus on the utility of certain models and ideas that can guide our acquisition and maintenance of clinical expertise. ${ }^{1-8}$ Understanding and applying these can, in turn, make us better clinician teachers and learners.

\section{A. How we think as experts:}

\section{A synopsis of models and mechanisms}

Dual process thinking is a commonly used theoretical model to explain the processes of development and practice of expert thinking and decision making. Rooted in cognitive psychology, the dual process model describes the synergistic interplay between two types of thinking: associative (also called non-analytical, intuitive, pattern recognition, or system 1) and analytical (also called system 2). ${ }^{9}{ }^{10}$ In turn, the dual process model has several components ${ }^{11}$ and understanding the development and relationships between these components may provide further insight into how we develop clinical diagnostic expertise in ourselves and our learners. (Figure 1)

\section{The components in the processes of clinical diagnostic expertise thinking}

Goethe said: "One sees what one knows". Certainly, a pre-requisite body of pertinent background knowledge and skills must be acquired by a novice in order that they may then selectively, flexibly and appropriately organize it around clinical findings and concepts. In essence, this development requires the transformation of background, dispersed knowledge into contextualized, practice-ready, compiled knowledge. The processes by which this is done include the identification and use of key clinical features -clinical attributes that help to rule in or rule out particular conditions or sets of diagnoses. ${ }^{8}$ For example, gender is a key feature in the approach to abdominal pain in the adult. Male gender immediately rules out several possibilities. A second complementary process is the development and use of meaningful (i.e. semantic) relationships between clinical

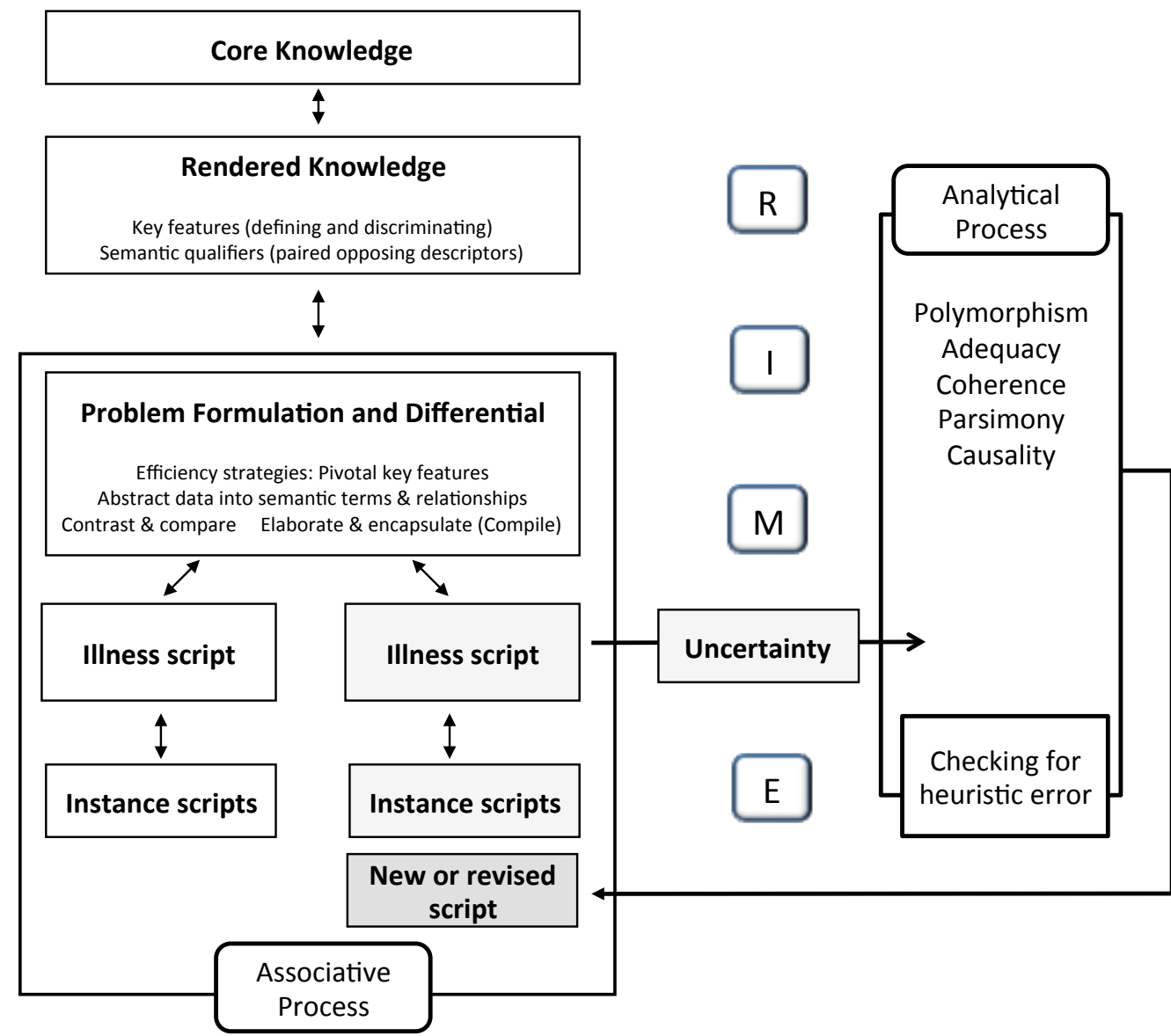

Figure 1. The dual process and components of clinical reasoning, with associated levels of RIME (reporter, interpreter, manager, educator) 
data or attributes. ${ }^{11}$ These can be used or recalled as clusters of information that help to organize thinking, and are building units for approaches and illness scripts (see below). Semantic qualifiers are used to abstract data into words or phrases that promote more rapid (and accurate) problem representation. ${ }^{11}$ Familiar examples are paired opposites such as acute versus chronic, unilateral versus bilateral, or mild versus severe. These are powerful tools for comparing and contrasting possible conditions. Observations of clinical experts thinking aloud suggest that successful diagnosticians use key features, semantic qualifiers and relationships earlier, more frequently, and in more diversified sets in their problem representations and discourses, than do less successful diagnosticians. ${ }^{12}$ Selfexplanation or diagnostic justification exercises also appear to be effective in helping medical students learn clinical reasoning. ${ }^{13,14}$ With practice, and experience, we learn which mix of key features, semantic qualifiers and relationships have the greatest utility in describing or discriminating given conditions or clinical problems.

\section{Developing dual processing in clinical diagnosis}

We can collate these condition-specific clusters of key features and relationships into prototypic representations called illness scripts. ${ }^{8}$ Related but more nuanced (i.e. polymorphic) presentations of the same condition are also learned and closely organized as instance scripts. The use of such scripts allows us to quickly look for only a subset of all the data that is available in a presenting condition, compare and contrast it to the features of our available scripts and determine a best match. This match is then compared to a larger portion of available data from the presenting condition. If there is concordance, this provisional match is deemed correct. Discordance prompts a search for a better match. The process continues until an acceptable match is perceived or uncertainty develops due to persistent discordance. Physicians refer to the mastery of this condition-specific process as learning an approach to a particular presenting condition, sign, or symptom. At the outset, learning is slow, conscious, and analytical, and requires support, either by preceptors or processes. Until key features and relationships are consolidated into the scripts, the novice's diagnostic approach typically uses too much non-contextual or superfluous case derived data. ${ }^{12}$ As the novice gains experience, the processes become automatic, unconscious, independent, and typified by efficient use of contextualized case-derived data. Unlike the analytical thinking mode that is prominent in the novice's diagnostic thinking, this associative mode appears to be used first, and most frequently, in expert diagnostic thinking. However, these dual modes of thinking remain entwined in a synergistic process at all stages of expert thinking development.

\section{Uncertainty and the interplay between associative and analytical thinking modes}

Uncertainty may occur when encountering novel situations (where the individual has no existing scripts) or when particular elements of the "match" between the presenting condition data, problem representation, and illness scripts remain discordant and require analysis. Uncertainty may also be triggered as part of internal or external stimulation of metacognitive processes (see below). Regardless, the perception of uncertainty characteristically causes an interruption in the associative thinking mode, or what John Dewey termed a perturbation. ${ }^{15}$

Ideally, this perturbation results in a switch into analytical thinking mode, characterized by slowing the diagnostic process and an explicit awareness of the process. At this point, responses may vary based on one's level of expertise and the clinical circumstances.

Novices may suspend the process and look for cognitive support in peers or preceptors. Expert clinicians, in busy or taxing clinical environments, may be tempted to ignore or perfunctorily dismiss the uncertainty. This is more likely if the clinician is tired, emotionally taxed, cognitively overloaded, or has perceived or real limitations of time. Since the clinical environment is never $100 \%$ certain, there is also a rationale for setting high thresholds for addressing uncertainty to prevent decision-making from becoming impractically slowed or even paralyzed. Even in relatively calm work environments, clinicians fail to pursue many clinical questions that arise, ${ }^{16}$ and this is likely true for explicitly addressing uncertainty. Unfortunately if this response becomes habitual, an unconscious and inappropriately high threshold for uncertainty may shut down clinical curiosity, prevent the development or maintenance of expertise, and risk slippage of expert thinking from a state of unconscious competence to one of unconscious incompetence.

More appropriately the uncertainty triggers an effortful analysis of the discordance. This often includes using more of the available data from the presenting condition, with the explicit assessment of "script match" for polymorphism, data adequacy, coherence, parsimony or even pathophysiological plausibility (see glossary for definitions). This analytical processing of uncertainty (also a learned skill) facilitates script development and importantly, the systematic exploration of errors and biases in data gathering, data abstraction, and decision-making. 


\section{Bias, error and the interplay between associative and analytical thinking}

The associative fast and frugal thinking mode employs shortcuts, by quickly matching problem representations to pre-constructed illness scripts. These work well most of the time, but the goal directed process of the shortcut (or heuristic) also creates an inherent capacity to generate error, called a bias. The heuristic and its bias(es) are inseparable. For instance, the heuristic "common things are common" is a familiar and time saving, reasoning strategy, as it allows for the rapid exploration of the probable. Unfortunately the availability bias incurred by expecting stereotypes may prevent the consideration of alternatives, and encourage premature closure in diagnostic thinking.

Despite this intrinsic potential for error, associative expert thinking has been shown to be at least as accurate as analytical thinking, as well as being faster. Proposed explanations include the impractical cognitive load on working memory posed by pure hypothetical-deductive approaches in real-time clinical reasoning, or the unrealistic Bayesian requirements for base rates of disease and diagnostic test characteristics that may not be available for particular clinical contexts. ${ }^{17}$ It is likely that the duality of the cognitive process is responsible: experts use both systems, and re-invest some of the thinking time saved using heuristics into analytically-based and effective methods of error checking. ${ }^{1,17}$ Thus the fast and frugal associative method, combined with an analytical check on uncertainty comes out ahead in both speed and accuracy.

\section{B. Teaching clinical diagnostic thinking through role modeling}

\section{Role modeling the dual process model}

The regular interplay between analytical and associative expert thinking, triggered by addressing uncertainty, may be how we develop new instance or illness scripts that are subsequently used in the associative mode. More than mere awareness of uncertainty, this triggering involves "thinking about thinking" or metacognition, and it is likely an essential element in the practice, development and maintenance of expertise, as described by Erickson and others. ${ }^{18}$ Triggering uncertainty and providing analytical cognitive support to promote habitual responses to this uncertainty can also develop, enhance, and maintain learners' clinical reasoning performance. The clinician-teacher role models this by demonstrating thinking aloud exercises (to patients, learners or colleagues), habitually undertaking diagnostic timeouts, making verbal summaries intermittently during clinical interviews, and consistently and explicitly addressing uncertainty and potential biases and errors in thinking. The process may initially involve heavy external scaffolding with explicit demonstration of the process, (medical schools and residencies), or, with growing independence, more infrequent and implicit cueing that evokes internalized analytical thinking protocols, such as "curbside" consultations with colleagues, second opinions or formal continuous professional learning.

Novices can thus develop or adopt component parts of the process, or even use borrowed approaches or scripts to conditions by exposure to (and emulation of) the behaviours modeled by more experienced clinicians. ${ }^{14,19}$ However, the development of competence and expertise in data abstraction, the use of key features and semantic qualifiers and relationships, problem representation, and the independent development and use of scripts, requires the individual experience of deciding, doing and showing, and the metacognitive reflection and assessment of the process. ${ }^{20}$ Clinician-teachers implicitly address this when they actively encourage novices to abstract, and relate, reported attributes such as the location, quality, severity chronology, setting, aggravating or alleviating factors, when discussing a patient's chief complaint or history of present illness. The novice learns to interpret "this morning" as acute onset, or 3 episodes of pain in the ankles, knees, hips, shoulders, elbows, wrists as "recurrent generalized large joint pain". Through observation and emulation of others and subsequent practice of clinical problem representations, they develop expertise in using linked key features, semantic qualifiers and meaningful relationships in their diagnostic decision making and discourse. Experienced clinician teachers can also (at least implicitly) assess and provide formative feedback for the development of learners' clinical diagnostic reasoning skills by attending to the use of these same components in oral case summaries or presentations. A learner's summary starting with: "This young previously healthy woman presents with acute dyspnea and hypoxia after prolonged bedrest from a back injury. She smokes and takes an oral contraceptive" constitutes evidence for a growing mastery of problem representation and a script to diagnostically approach the condition at hand.

\section{Using the one minute preceptor and RIME}

Observation and assessment of clinical reasoning may be enhanced by asking learners to give a verbal summary using the one-minute preceptor (OMP) format. ${ }^{21}$ First, the learner makes a commitment by stating what they think is happening (i.e., diagnosis or condition), and then explains their underlying reasoning using linked key features, and semantic qualifiers and relationships and problem representation. The preceptor provides feedback and scaffolding to help the learner 
assess and reflect on their performance and decide what performance elements may be consolidated and which need work, and provides general rules that can help revise and build new illness and instances scripts.

RIME is an acronym for a commonly used schemata that describes the stages of performance from novice to expert in clinical communication, reasoning and decisionmaking skills. ${ }^{22}$ These stages include the reporter, interpreter, manager and educator (or expert). Clinician-teachers can use RIME when observing learner performance to assess levels of mastery in clinical reasoning and decision making, and to guide consolidative and constructive feedback. If learners are also taught to use RIME and OMP, they may internalize these frameworks to act as both metacognitive triggering tools and as subsequent frameworks for the independent assessment of their own clinical reasoning performance. For example, learners who intermittently summarize their abstraction of collected data in an interview, and then ask themselves: "Do I now have an idea of what is going on?" (step one of OMP, the educator in RIME), may respond to "no" by determining whether it is the result of a lack of scripts or experience with the condition (manager), inadequate or incorrect abstraction of data or problem representation (interpreter), or a basic lack of compiled (or even basic) knowledge required to approach this condition (reporter). This allows for more directed activity to improve performance.

\section{Continuous professional learning by role modeling and teaching clinical diagnostic thinking}

Unfortunately, once clinicians are in independent practice, the support for development and maintenance of our clinical diagnostic expertise diminishes. The task of sustaining high levels of meta-cognitive activity on one's own can be difficult, if not impossible. It is least likely to occur in circumstances that are the most cognitively-taxing (and error prone), and in areas of expertise that use associative, unconscious thinking. Hence we are at risk of sliding from unconscious competence into unconscious incompetence. Therefore even the most experienced clinicians need a coach at some point. Using undergraduate or postgraduate training models of preceptorship to provide this may be impractical owing to cost and availability, and the present culture of clinical practice does not include regular academic detailing by peers or preceptors for continuous practice improvement.

Physicians who teach medical students and residents believe teaching is a powerful professional development stimulus for their own learning and maintenance of competence. $^{23}$ As clinician-teachers, we need to deconstruct our unconscious competence (expertise) into conscious competence (i.e., mastery) in order to explain, clarify, and explore steps in reasoning with learners and to assess their performance by comparison to our own clinical reasoning. This results in frequent critical appraisal of our own clinical diagnostic reasoning, and opportunities to learn new scripts and alternative strategies from others. The process of teaching becomes a triggering event for the teacher's own deliberate practice. This can be a particularly effective if observations and discourse of teachers and learners are guided and focused by shared and explicit use of schemata such as OMP and RIME. To paraphrase Aristotle, clinical teaching may also be the highest form of continuous professional learning, and it may also be the most practical, economic, and sustainable method for the maintenance of clinical diagnostic expertise.

\section{References}

1. Elstein AS. Thinking about diagnostic thinking: a 30-year perspective. Adv in Health Sci Educ 2009;14:7-18.

2. Epstein RM Mindful practice JAMA 1999;282:833-9.

3. Eva KW. What every teacher needs to know about clinical reasoning. Med Educ 2005;39:98-106.

4. Kulasegaram KM, Grierson LE, Norman GR. The roles of deliberate practice and innate ability in developing expertise: evidence and implications. Med Educ 2013;47(10) 979-989

5. Moulton CE, Regehr G, Mylopoulos M et al. Slowing down when you should: a new model of expert judgement. Acad Med 2007; 82(suppl): 109-17.

6. Norman G, Young M, Brooks L. Non-analytical models of clinical reasoning: the role of experience. Med Educ 2007;41: 1140-1145.Redelmeir DA, Ferris LE, Tu JV, et al. Problems for clinical judgment: introducing cognitive psychology as one more basic science. CMAJ 2001;164: 358-360.

7. Schmidt H Rikers R. How expertise develops in medicine: knowledge encapsulation and illness script formulation. Med Educ 2007;41:1133-9.

8. Charlin B, Boshuizen HPA, Custers EJ, Feltovich PJ. Scripts and clinical reasoning. Med Educ 2007: 41: 1178-1184

9. Norman GR, Eva KW. Diagnostic error and clinical reasoning. Med Educ 2010;44:94-100.

10.Bordage G. Prototypes and semantic qualifiers: from past to present. Med Educ 2007;41: 1117-1121

11.Bordage G. Elaborated knowledge: A key to successful diagnostic thinking. Acad Med 1994;69:883-885.

12. Nendaz MR, Bordage G. Promoting diagnostic problem representation. Med Educ 2002;36:760 -766.

13. Chamberland M, Mamede S, St-Onge C. Self-explanation in learning clinical reasoning: the added value of examples and prompts. Med Educ 2015; 49: 193-202.

14.Cianciolo AT, Williams RG, Klamen DL, et al. Biomedical knowledge, clinical cognition and diagnostic justification: a structural equation model. Med Educ 2013;47:309-316.

15. Boydston A (Ed.). John Dewey: The Later Works, 1925-1953 (Carbondale and Edwardsville: Southern Illinois Press, 1987).

16.Del Fiol G; Workman TE; Gorman PN. Clinical questions raised by clinicians at the point of care: a systematic review. JAMA Internal Medicine. 2014; 174(5):710-18.

17.Wegwarth O, Gaissmaier W, Gigerenzer G. Smart strategies for doctors and doctors-in-training: heuristics in medicine. Med Educ;2009;43: 721-728

18. Ericsson KA. An expert-performance perspective of research on medical expertise: The study of clinical performance. Med Educ 2007; 41:1124-1130. 
19.Ericsson KA. Deliberate practice and the acquisition and -maintenance of expert performance in medicine and related domains. Acad Med 2004;79: S70-81.

20.Kassirer J. Teaching clinical reasoning: Case-based and coached. Acad Med 2010;85: 1118-1124.

21. Alguire PC, DeWitt DE, Pinsky LE. Teaching in your office: a guide to instructing medical students and residents. American College of Physicians 2001. Philadelphia, PN

22.Pangaro L. A new vocabulary and other innovations for improving descriptive in-training evaluations. Acad Med 1999;74: 41-5.

23. Wenrich MD, Jackson MB, Ajam KS, et al.Teachers as learners: The effect of bedside teaching on the clinical skills of clinician-teachers. Acad Med 2011; 86(7): 846-852.

\section{Glossary of Clinical Reasoning Terminology}

\section{Analytical reasoning}

- Deliberate, studied, problem solving process that consciously and mindfully uses rules of diagnosis and compares the signs and symptoms of the presenting case against them. It uses knowledge of causal mechanisms, and symptombased probabilities, and typically carefully outlines all clinical features before generating any diagnostic hypotheses. Other terms include hypo-deductive or Bayesian reasoning.

\section{Adequacy}

- An analytical strategy component that helps assess the likelihood of a diagnosis being correct on the basis of whether all of the findings are explained by it.

\section{Causality}

- An analytical strategy component that involves forming inferences based on major cause-and-effect relations between clinical variables or events. Such reasoning often relies on the pathophysiologic aspects of individual disease states, so its application is far narrower diagnostically than the other analytic strategy components. However, it may be very helpful in analyzing discrepancies in certain diagnoses, and help assess disease polymorphism (see below).

\section{Coherence}

- An analytical strategy component that is partly a corollary of adequacy (see above). It helps assess the likelihood of a diagnosis being correct by assessing if the key features link together, and if in turn they are linked to the diagnosis.

\section{Clinical reasoning}

- A complex process that uses cognition, metacognition, discipline-specific knowledge, and context to gather and analyze patient information, evaluate its significance, and weigh alternative conclusions or actions.

\section{Complied (encapsulated) summaries}

- Summaries that are semantically rich and selective in the data presented. They are characterized by selectively presented data that is held together by numerous semantic associations. The "thesis" or train of thought is focussed and easily followed and cogent. Associations are readily identifiable, and coherently and plausibly linked.

\section{Disease polymorphism}

- An analytical strategy component that helps assess the likelihood of a diagnosis being correct in situations where the patient's clinical manifestations fail to precisely match the textbook or classic description of a disease state.

\section{Elaboration}

- A behavior composed of rendering patient derived data into identifiable key features (see below), using semantic qualifiers (see below), and developing semantic associations or networks of relationships between these clusters of qualifiers and key features. Hence a 5 minute episode of face and arm weakness becomes transient (versus fixed), unilateral (versus bilateral or localized), and upper (versus lower) motor neuron pattern. Problem representations or summaries that demonstrate elaboration include more extensive syntactic definition of findings, with greater use of semantic qualifiers, networks or clusters of findings (semantically rich, extended discourses). Elaboration becomes expertise when it is selective (addresses pertinent key features) and plausibly and parsimoniously linked to a diagnosis (compilation)

\section{Heuristic (error)}

- A simple decision making strategy that allows for solving problems and making judgments quickly and efficiently; a Rule-of-thumb or algorithm for reasoning

\section{Illness script methodology}

- Exemplar based knowledge of a condition experientially developed and used in intuitive (non-analytical) clinical reasoning strategies. It is characterized by first impressions and quick pattern recognition drawing on episodic memory and rapid response to presented information. An example would be the rapid appreciation of a typical presentation and natural history of a pulmonary embolism. This is also called similarity based reasoning.

\section{Instance script methodology}

- A nuance or conditional subset of an illness script. For example, the patient with a pulmonary embolism that presents as an episode of syncope with no evidence for hypoxia or other bedside clinical findings. Instance scripts may be derived from the analytical experience of repetitively determining the range of variability in a condition's presentation. (See disease polymorphism)

\section{Associative Clinical reasoning (see illness script methodology)}

- This process is primed to recognize new situations or patterns in its rapid recall fashion after repeated exposure to the same stimuli or set of events. Associative components often produce valuable, accurate responses, but because of their inherent characteristics of quickness and apparent lack of computation), they can be influenced by the context of the moment, and are prone to heuristic error. Synonyms include non-analytic, intuitive, tacit, pattern recognition, similarity based, "illness or instance scripts-based" or system 1 reasoning strategies.

\section{Key features}

- Defining and or discriminating features of particular conditions or clinical presentations, useful (either individually or in groups) for "ruling in" or "ruling out" diagnoses. Key features that have powerful discrimination or defining characteristics for particular conditions are often included in "efficiency strategies" to quickly render down the number of diagnostic possibilities. For example, determining if the patient presenting to the emergency department with acute abdominal pain is a male can quickly rule out a number of diagnostic possibilities including complications of pregnancies, pelvic inflammatory disease and ectopic pregnancy.

\section{Meta-cognition}

- An individual's knowledge, awareness and command of thinking and learning strategies. A method of introspection in which one contemplates or reflects on one's own thinking.

\section{Parsimony}

- An analytical strategy component that helps assess the likelihood of a diagnosis being correct on the basis of whether it is the simplest explanation for the constellation of findings/case presentation. Often referenced to Occam's razor.

\section{Semantic qualifiers}

- Terms that are abstractions of the patient's description of their symptoms and detected signs. They are paired and oppositional in nature and are used to compare and contrast findings and diagnostic considerations. For example, "one year of symptoms" becomes "chronic" (versus acute) and rash only on the leg becomes "localized" (versus generalized). Semantic qualifiers add meaning to features and are more easily combined as clusters of such dichotomous qualifiers to form meaningful relationships. Successful diagnosticians use semantic qualifiers more frequently and in more diversified sets (or networks) in their discourses than diagnosticians who are less successful. 